
\title{
3 Research Suare \\ The Effect of Parents 'web-based training on the Level of Post-Traumatic Stress Symptoms in Children: A Quasi-Experimental Study
}

\section{Zakieh Omidvar Ashkalak}

Department of Pediatric nursing, faculty of nursing and midwifery, Iran University of Medical Sciences Hadis Nazari

School of nursing and midwifery, llam University of Medical Sciences

\section{Naima Seyedfatemi}

Department of psychiatric nursing, faculty of nursing and midwiferylran University of Medical Sciences Hamid Haghani

Department of Biostatistics, Iran University of medical sciences

Soroor Parvizy (DS_parvizy@yahoo.com )

\section{Research article}

Keywords: Stress Disorders, Post-Traumatic, Web-based-learning, parents, child

Posted Date: March 16th, 2020

DOI: https://doi.org/10.21203/rs.3.rs-17354/v1

License: (c) (1) This work is licensed under a Creative Commons Attribution 4.0 International License. Read Full License 


\section{Abstract}

\section{Background}

Post-traumatic stress disorder is a common occurrence in children that can affect their overall physical and mental functioning and quality of life. Early intervention can have significant health benefits for children. The purpose of this study was to investigate the effects of parents' web-based training on the level of post-traumatic stress symptoms in children.

\section{Methods}

This study was a quasi-experimental one with two groups that is intervention and control group. 110 children aged 10 to 18 with traumatic stress symptoms and one of their parents were selected through available sampling. Data were collected by a researcher-made demographic questionnaire and the Child Revised Impact of Events Scale (CRIES-8). The control group received routine care, but the experimental group received a 4-week training course through a researcher-designed website. Two weeks after the intervention, the child stress level was measured and compared within both groups. Data were analyzed using SPSS V.22.

Results

The difference between the mean score of total traumatic stress and its subscales before intervention was not statistically significant $(P=0.23)$. After intervention, however, the mean score of total traumatic stress and its subscales decreased in the experimental group and increased in the control group and the difference was statistically significant $(P<0.001)$.

\section{Conclusions}

E-learning intervention by parents as a nursing intervention reduces the level of traumatic stress symptoms in children. This easy task of web-based training can be recommended to reduce the symptoms of traumatic stress and possibly to increase recovery in these patients.

\section{Background}

Accidents are one of the most important causes of hospital admissions in children. PTSD (PostTraumatic Stress Disorder) is one of the commonest mental disorders in children who have had injuries. Most children and their parents have reported at least one severe post-traumatic stress disorder reaction during the first month after a traumatic event $(1,2)$; this disorder is much more common in children than in adults (3). A recent meta-analysis reported the prevalence of PTSD in children to be $15.9 \%$ (4). Also in the Cohort study, $31.1 \%$ of children up to the age of 18 experienced trauma, of which $7.8 \%$ had undergone PTSD (5). 
PTSD is strongly associated with behavioral and emotional, interpersonal problems, drug abuse, academic failure, sleep disorders, depression, anxiety, ADHD (Attention Deficit Hyperactivity Disorder) and impaired quality of life. These problems are amongst major risks to the society and increase the risk of developing chronic stress-related physical illnesses, as a result, identification and treatment of PTSD children is of vital importance $(1,6)$.

Recently, more efforts have focused on secondary prevention of existing symptoms through early intervention. Secondary prevention efforts are performed immediately after the trauma (less than 6 weeks after the trauma), and its intensity and concentration are dependent on the child's risk status and the level of his need. Early preventive interventions during the first few days of symptom onset both reduce and prevent acute PTSS (Post-Traumatic Stress Symptoms) and prevent the onset of chronic PTSD (7).

Parents are a major source of support for children after an injury (8); and based on the results of mental studies, the extent of their discomfort following a child trauma, affected children's ability to adjust emotionally and control pain or trauma. Parental response to a traumatic event in a child plays an important role in the psychological recovery of the child after an accident (9). In addition, maladaptive family functioning is one of the causes of PTSD in children after experiencing a traumatic event (10). On the other hand, parents are extremely weak in identifying acute PTSS in their children (8).

Training the patient and his / her family is an important task and is one of the quality standards of nursing care (11). One of the major concerns of pediatric nurse practitioners is to promote the health of the child both physically and mentally (12); This can be accomplished by training the client and her family in family-centered care, however, problems such as lack of time, insufficient resources and expertise have always prevented effective clinical training. Rapid advances in the internet access are not only paving the way for a dynamic mode of delivering psychological empowerment interventions, but can also be widely disseminated and implemented. Online training allows for standardization of the content of the program, can be tailored to specific needs of age groups and evolutionary stages, as well as social interaction and can also be easily updated (13).

Based on the results of the studies, Internet-based psychosocial education interventions have been effective for a wide range of populations with physical and psychiatric problems, including the PTSD population and parents of children with trauma; In parents and children (8), Prevents stable and chronic PTSD in children (14). In addition, the provision of an Internet-based psychological support program for caregivers of children with cystic fibrosis has shown reduced anxiety and depression and improved parental quality of life (15). In another study, intervention using Internet feedback has been shown to improve children's growth and reduce their obesity (16). But, in Iran, as far as we know, research into interventions for PTSD in children has only paid to the behavioral therapy (17), play therapy (18) or desensitization (19) for the child, while parents, as the primary focus of child life, have been overlooked in studies related to this topic. Also, none of these studies have provided Internet-based training; The purpose of this study was to investigate the effects of parents' web-based training on the level of posttraumatic stress symptoms in children. 


\section{Methods}

This is a Clinical trial with control and intervention group, non-randomized (quasi-experimental), non-blind study. The study was carried out at Shahid Rajaee Hospital in Qazvin, Iran. The participants of the study consisted of all traumatized children and one of their parents (the father or mother who had more contact and provided much care for the child).

Sample size was determined at $95 \%$ confidence level with $80 \%$ test power and 102 participants (51 in each group). Finally, a total of 110 individuals were included in the study using available sampling method from 06 march 2018 to 21 April 2018. In order to prevent mixing of samples and data contamination, a 15- day interval between selection of control and intervention group was considered, as a result, 15 days after discharge of last sample of control group, the intervention group was sampled.

The participants' inclusion criteria were as follows: age range of 10-18, hospitalization of a child due to a traumatic accident including crashes, car and motor vehicle accidents, limb fractures, multiple injuries, limb injuries, vein injuries, visceral injuries, burns and mild head injuries (GCS: Glasgow Coma Scale >14), child hospitalization 3 days to one-month post-traumatic injury, score above 17 on the CRIES-8 questionnaire, and no evidence of parental abuse due to child injury. Parents' inclusion criteria were: the ability to read and write in Persian, holding a high school diploma, the ability to use the internet, surf the web and handle electronic devices and the possession of no known mental or physical impairment. Exclusion criteria included: child death during the study, GCS decline, and change in child's level of consciousness after entering the study and unwillingness to the continuation of the study.

The instrument used in this study was a two-part questionnaire. The first part was a researcher-made demographic questionnaire that developed based on the literature review and under the supervision of pediatric nursing and psychiatric experts and included 12 questions about child and parent demographic information, parental access to the Internet, and their ability to use the internet; and the second part was the CRIES-8, which has 8 questions and two subscales; designed for use on children aged 8 years and older who are able to read independently and include 4 questions to measure Intrusion subscale and 4 questions to avoidance subscale are hence called it CRIES-8. Each question is scored using a 4-point Likert scale of zero (never) to 5 (most of the time) and the total score is obtained by summing the scores of each question. A score above 17 indicates a child with $\operatorname{PTSD}(20,21)$.

To determine the validity, the questionnaires were distributed to 10 nursing faculty members and experts in psychiatric and pediatric nursing. In order to determine the reliability using Cronbach's alpha method, the total correlation coefficient was 0.91 and 0.8 and 0.65 for each of the avoidance and intrusion subscales, respectively.

The researcher referred to the research environment. The researcher first prepared a list of eligible patients using patient records and information obtained from nurses in the ward, and after explaining their study goals and their willingness to accept or reject participation with informed consent and written consent questionnaires, the participants were asked to complete a demographic questionnaire by a 
parent and the CRIES-8 questionnaire by a child. After determining the samples based on inclusion criteria, these questionnaires were considered as pre-test. The questionnaires were coded after completion. The participants were assured that their personal information and responses would remain confidential. The control group received no intervention other than the routine care provided by the research setting, but the intervention group received a four-week child and parent stress training course and psychotherapy online via the researcher website (www. PTSD- pediatric-nursing-care.ir). The educational content of the website was compiled and completed by the researcher and finally designed by a computer engineer under the supervision of the researcher. The website content included topics related to the definition of PTSD, its symptoms in the child, and how to treat the child and help him/her to improve mental health. After completing the design of website, in order to determine the content validity and final approval, it was reviewed by IT experts, pediatric and psychiatric nursing team members, as well as by an emergency medicine physician and a child psychologist; after that supplementary comments were applied to the structure and content of the site. The intervention group were trained for 4 weeks and then for two weeks the study samples were given time to influence the intervention. After the sixth week, the level of child stress was again measured by CRIES-8 in two groups and the results were compared. Post-test data were collected by the researcher through telephone, e-mail and, in some cases, in person.

Data were analyzed using descriptive (frequency, mean) and inferential statistics (chi-square, independent t-test, paired t-test) in SPSS V.22 software. Significance level was considered $p<0.05$.

\section{Results}

During the study, two participants from the control group and three from the intervention group were excluded from the intervention due to parental withdrawal and lack of cooperation in completing the posttest questionnaire, thus reducing the total number of participants to 105 at the end of the intervention (53 patients in control group and 52 in experimental group.) The majority of children (53.3\%) were girls. The mean age in the control group was $13.15 \pm 2.29$ and in the intervention group was $13.28 \pm 2.38$. most frequent and the three main causes of injuries were vehicle and motor vehicle accidents (32.9\%), limb fractures $(17 \%)$ and crashes $(15.4 \%)$ in the intervention group, and the car and motorcycle accidents (28\%), burns (18/95) and limb fractures $(17 \%)$ in the control group. In both groups, the majority of parents $(>50 \%)$ had a high school diploma; the majority of fathers and mothers were employed (89.5\%) and were housewives (58\%), respectively. In terms of economic status, the majority of the experimental group had moderate (53.8\%) status and the majority of the control group had good status (50.9\%). the majority of parents In the experimental group, were better at surfing the web (48.1\%) and the control group were skilled at working with email (60.3\%).

The two groups were similar in terms of demographic characteristics and there was no statistically significant difference between them.

In the study objectives, as Table 1 shows, based on independent t-test, the difference between the mean, total stress score after the accident $(p=0.23)$ and the subscales of intrusion $(p=0.42)$ and avoidance $(p$ 
$=0.17)$. There was no significant difference between the two groups before the study, but after intervention, the mean score of total PTSD and its subscales decreased in the experimental group compared to the control group, and the difference between the two groups was statistically significant ( $p$ $<0.001)$.

Paired t-test results also showed that in the control group the mean score of total PTSD and its subscales after intervention increased and this difference was statistically significant $(p<0.001)$. Also based on the results of the same test in the experimental group the mean score of total PTSD and its subscales after the intervention were lower than prior to the intervention and this difference was statistically significant $(p<0.001)$.

Table 1

Comparison of the mean of total PTSD score and its subscales before and after the intervention in both groups.

\begin{tabular}{|c|c|c|c|c|}
\hline Subscales & Time & $\begin{array}{l}\text { Intervention group } \\
\text { mean } \pm S D\end{array}$ & $\begin{array}{l}\text { Control group } \\
\text { mean } \pm \text { SD }\end{array}$ & P-value \\
\hline Intrusion & $\begin{array}{l}\text { Before education } \\
\text { After education } \\
\text { P-value a }\end{array}$ & $\begin{array}{l}12.15 \pm 3.28 \\
8.9 \pm 3.2 \\
<0.001\end{array}$ & $\begin{array}{l}11.64 \pm 3.31 \\
14.33 \pm 3.65 \\
<0.001\end{array}$ & $\begin{array}{l}0.42 \\
<0.001\end{array}$ \\
\hline Avoidance & $\begin{array}{l}\text { Before education } \\
\text { After education } \\
\text { P-value }\end{array}$ & $\begin{array}{l}12.76 \pm 3.43 \\
10.42 \pm 2.66 \\
<0.001\end{array}$ & $\begin{array}{l}11.92 \pm 2.93 \\
14.13 \pm 3.41 \\
<0.001\end{array}$ & $\begin{array}{l}0.17 \\
<0.001\end{array}$ \\
\hline Total score & $\begin{array}{l}\text { Before education } \\
\text { After education } \\
\text { P-value }\end{array}$ & $\begin{array}{l}24.92 \pm 6.05 \\
19.32 \pm 5.18 \\
<0.001\end{array}$ & $\begin{array}{l}23.56 \pm 5.56 \\
28.47 \pm 6.51 \\
<0.001\end{array}$ & $\begin{array}{l}0.23 \\
<0.001\end{array}$ \\
\hline \multicolumn{5}{|c|}{ a. paired t-test } \\
\hline \multicolumn{5}{|c|}{ b. independent t-test } \\
\hline
\end{tabular}

\section{Discussion}

According to the results of the present study, the commonest type of accidents that children experienced were car or motor vehicle accidents, with approximately $30 \%$ of the sample being in car accidents, which is significant percentage. However, as the results of the study indicated, the majority of participants aged 10 to 13, in this age group, most accidents involving children are car accidents (12). But parental neglect to take simple steps that can reduce vehicle injuries to children, such as using a seat belt, not seating children under 13 in the front seat and using a child restraint (12), as well as failure in the instruction of the traffic signs to help children cross the street and, the lack of strategies and laws that protect children from such injuries can be some possible reasons; as a meta-analysis study showed that almost half of children with PTSD experienced non interpersonal accidents, such as a motor vehicle accident, life- 
threatening illnesses, or loss of loved ones (4). So training about these causes created by school health nurses for children as well as their parents can be helpful and prevent child injuries.

The results of this study showed that web-based teaching and training to parents reduce PTSS in their children, as in the control group that received routine care in the study environment, their PTSS increased and this increase was statistically significant $(p<0.001)$. Therefore, it can be concluded that there is no intervention or intervention in the hospital to reduce PTSD in children are ineffective, which may be due to the lack of awareness of the nurses or lack of sufficient time for education; On the other hand, due to the harmful effects that PTSD can have on children, it is important to have effective interventions in this area. Therefore, using web-based training for parents can be beneficial as well as reducing the time spent by nurses to perform such trainings. It is available and low cost as well.

The results of this study are consistent with several studies demonstrating the importance and usefulness of e-learning for children or parents in the field of PTSD. The study that was most consistent with the present study was research which showed that e-learning for parents of children aged 6-17 with PTSS using the web is feasible, leading to increased parental knowledge and reduced PTSS in children and their parents. However, these effects were only immediately after the intervention and there was no statistically significant difference between the two groups in the follow-up 6 weeks after the intervention (8). In the present study, two weeks after the end of the intervention, a significant reduction in PTSS in children was shown, which could be due to a shorter intervention time in the Marsac and colleague's study (20 minutes), whereas two weeks was considered as intervention time in this study. Another similar study suggests that early intervention in the early days after a traumatic event and when the child is still hospitalized web-based training, using attractive online games for children aged 8-12, can prevent permanent PTSD in children (7). Also, in another study, web-based intervention for parents of children with burns and with the aim of reducing PTSD and general stress in parents only a short time after the intervention, immediately after and 3 months after the intervention, was effective in reducing PTSD in the parents. There was no statistically significant difference between the experimental and control groups in PTSD at 12-month follow-up (22).

In the present study, two weeks after the intervention, the PTSD symptom decreased in the children of the experimental group, but we don't have the follow-up period for the intervention; therefore, it seems that parental training alone did not have a significant effect or use of longer training time for them may be helpful. Another similar study examining the impact of e-learning is a study in which e-feedback to parents helped children grow and reduce their obesity (16). Also web-based and electronic support for parents of children with cystic fibrosis have also led to reduced anxiety and depression and improved quality of life in parents (15); And e-learning for adolescents aged 11 to 14 with diabetes led to increased quality of life and decreased HbA1c (Hemoglobin A1c) in them (13).

One of the limitations of the present study was to talk to parents about filling out a questionnaire during stressful emergency procedures in the emergency department. For this purpose, it was tried not to talk to parents immediately upon arrival to the emergency room and they were asked to complete the 
questionnaire after resolving their concerns and moving the child to the ward. Also, how children complete the post-test questionnaire at home, and whether the questionnaire was completed by the child or parents may influence the study outcomes which are beyond the control of the researcher, However, attempts were made to alleviate some of these limitations by emphasizing parents to complete the questionnaire by the child themselves as well. Another limitation of the study was that there was no strategy for monitoring the intervention group in terms of web surfing time period and the use. Patients in this study were selected from Shahid Rajaee Hospital in Qazvin; and sampling was done on a continuous and non-random basis, so the generalizations of the present study should be taken with caution.

Therefore, it is recommended to conduct more studies with larger sample size and longer follow-up periods ( 6 months intervals) to evaluate its long-term effects and the likelihood of children with PTSD in the future. In addition, given that this study was conducted in Qazvin, it is suggested that the present study be conducted on children in other cities as well. It is also suggested to conduct a study comparing child and parent training. In addition, it is useful to conduct a study that examines the knowledge and attitudes of pediatric nurses about PTSD as well as measures taken in hospitals to prevent PTSD in children.

\section{Conclusions}

In this study, the findings showed that e-learning interventions to parents of traumatic children reduce the level of post-traumatic stress symptoms in children. Nurses as members of the health team, play a key role in maintaining and promoting community health by playing roles such as counselor, supporter and trainer in prevention and rehabilitation, they can utilize different educational processes to increase awareness of clients in Promote self-care and use of measures to prevent the spread of disease in the community (11). Therefore, internet based training is recommended as an easy, accessible and low cost way to reduce the symptoms of traumatic stress and possibly increase recovery in such groups of patients for nursing use.

\section{Abbreviations}

PTSD: Post Traumatic Stress Disorder IRCT: Iran Registry of Clinical Trial; ADHD: Attention Deficit Hyperactivity Disorder; PTSS: Post Traumatic Stress Symptom; GCS: Glasgow Coma Scale; CRIES: Child Revised Impact of Events Scale; HbA1c: Hemoglobin A1c.

\section{Declarations}

\section{Ethics approval and consent to participate}

After explaining the purpose of the study and being free to accept or reject the study, written informed consent was obtained from parents and children. A written informed consent was also obtained from 
parents for children under the age of 16 years old. Ethics have been complied with and approved by the Ethics Committee of Iran University of Medical Sciences (IR.IUMS.FMD.REC1396.9211196239).

\section{Acknowledgment}

The authors need to express their gratitude to all the staff and personnel of Shahid Rajaee Hospital in Qazvin who provided the necessary cooperation.

\section{Author's contributions}

Researcher ZOA: developed the specific study idea, study design, data gathering, HN: Concept development, study design, preparation the article draft, NS: Concept development, study design, $\mathrm{HH}$ : study design, Statistical and data analysis, preparation the article draft, SP: Project leader, Concept development, study design, preparation the article draft, final revision of article. All authors read and approved the final manuscript.

\section{Funding}

This article has been funded by the Research Vice President of Iran University of Medical Sciences.

\section{Availability of data and materials}

The data used and analyzed during the current study are available from the corresponding author upon reasonable request.

\section{Consent for publication}

Not applicable for this study.

\section{Competing interests}

the authors declare no competing interests.

\section{References}

1. Zhang Y, Zhou X, Yang L, Hetrick SE, Weisz JR, Cuijpers P, et al. Comparative efficacy and acceptability of psychotherapies for post-traumatic stress disorder in children and adolescents: study protocol for a systematic review and network meta-analysis. BMJ open. 2018;8(3):e020198.

2. Marsac ML, Kassam-Adams N, Hildenbrand AK, Nicholls E, Winston FK, Leff SS, et al. Implementing a Trauma-Informed Approach in Pediatric Health Care Networks. JAMA pediatrics. 2016;170(1):70-7.

3. Kovachy B, O'Hara R, Hawkins N, Gershon A, Primeau MM, Madej J, et al. Sleep disturbance in pediatric PTSD: current findings and future directions. Journal of clinical sleep medicine : JCSM : official publication of the American Academy of Sleep Medicine. 2013;9(5):501-10. 
4. Alisic E, Zalta AK, van Wesel F, Larsen SE, Hafstad GS, Hassanpour K, et al. Rates of post-traumatic stress disorder in trauma-exposed children and adolescents: meta-analysis. The British journal of psychiatry : the journal of mental science. 2014;204:335-40.

5. Lewis S, Arseneault L, Caspi A, Fisher H, Matthews T, Moffitt T, et al. The epidemiology of trauma and post-traumatic stress disorder in a representative cohort of young people in England and Wales. The lancet Psychiatry. 2019;6:247-56.

6. Salari R, Malekian C, Linck L, Kristiansson R, Sarkadi A. Screening for PTSD symptoms in unaccompanied refugee minors: a test of the CRIES-8 questionnaire in routine care. Scandinavian journal of public health. 2017;45(6):605-11.

7. Kassam-Adams N, Marsac ML, Kohser KL, Kenardy J, March S, Winston FK. Pilot Randomized Controlled Trial of a Novel Web-Based Intervention to Prevent Posttraumatic Stress in Children Following Medical Events. Journal of pediatric psychology. 2015;41(1):138-48.

8. Marsac ML, Hildenbrand AK, Kohser KL, Winston FK, Li Y, Kassam-Adams N. Preventing posttraumatic stress following pediatric injury: a randomized controlled trial of a web-based psychoeducational intervention for parents. Journal of pediatric psychology. 2013;38(10):1101-11.

9. De Young AC, Hendrikz J, Kenardy JA, Cobham VE, Kimble RM. Prospective evaluation of parent distress following pediatric burns and identification of risk factors for young child and parent posttraumatic stress disorder. Journal of child and adolescent psychopharmacology. 2014;24(1):917.

10. Dorrington S, Zavos H, Ball H, McGuffin P, Sumathipala A, Siribaddana S, et al. Family functioning, trauma exposure and PTSD: A cross sectional study. Journal of affective disorders. 2019;245:64552.

11. Dehghani a, orang $m$, abdollahyfard s, parviniyan nasab am, vejdani ma. Barriers to Patient Education in Clinical Care Viewpoints of Nurses. Iranian Journal of Medical Education. 2014;14(4):332-41.

12. Hockenberry M, Rodgers C, Wilson D. Wong's Essentials of Pediatric Nursing 10th ed: Elsevier Mosby; 2016.

13. Grey M, Whittemore R, Jeon S, Murphy K, Faulkner MS, Delamater A. Internet psycho-education programs improve outcomes in youth with type 1 diabetes. Diabetes care. 2013;36(9):2475-82.

14. Kenardy JA, Cox CM, Brown FL. A Web-Based Early Intervention Can Prevent Long-Term PTS Reactions in Children With High Initial Distress Following Accidental Injury. Journal of traumatic stress. 2015;28(4):366-9.

15. Fidika A, Herle M, Lehmann C, Weiss C, Knaevelsrud C, Goldbeck L. A web-based psychological support program for caregivers of children with cystic fibrosis: a pilot study. Health and quality of life outcomes. 2015;13:11.

16. Dam R, Robinson HA, Vince-Cain S, Heaton G, Greenstein A, Sperrin M, et al. Engaging parents using web-based feedback on child growth to reduce childhood obesity: a mixed methods study. BMC public health. 2019;19(1):300. 
17. Mahmoudi-Gharaei J, Mohammadi M, Bina M, Yasami M, Fakour Y, Naderi F. Behavioral group therapy effect on Bam earthquake related PTSD symptoms in children: A randomized clinical trial Iran J Pediatr. 2007;16(1):-

18. Shamsi pour A, Solgi R, Rozbahani M, Babaee Amirir N, Darabi B. Effectiveness of of Play Therapy (with Sand Play Approach) in Children with PTSD. Journal of Exceptional Children. 2019;18(4):55-66.

19. Moghadam AH, Sharbaf HAM, Mashhadi A. Effectiveness of Eye Movement Desensitization and Reprocessing(EMDR) to Reduce the Severity of Symptoms of Post-Traumatic Stress Disorder and Stuttering With Psychological Origin(Case Study in Child with Four Years Old). Arak Medical University Journal. 2017;19(11):87-98.

20. Verlinden E, van Meijel EP, Opmeer BC, Beer R, de Roos C, Bicanic IA, et al. Characteristics of the Children's Revised Impact of Event Scale in a clinically referred Dutch sample. Journal of traumatic stress. 2014;27(3):338-44.

21. Perrin S, Meiser-Stedman R, Smith P. The Children's Revised Impact of Event Scale (CRIES): validity as a screening instrument for PTSD. Behavioural and Cognitive Psychotherapy. 2005;33(4):487-98.

22. Sveen J, Andersson G, Buhrman B, Sjoberg F, Willebrand M. Internet-based information and support program for parents of children with burns: A randomized controlled trial. Burns : journal of the International Society for Burn Injuries. 2017;43(3):583-91.

\section{Supplementary Files}

This is a list of supplementary files associated with this preprint. Click to download.

- CONSORT2010Checklist.doc 